\title{
Atypical Presentation of Lumbosacral Spinal Cord Epidural Abscess: A Case Report
}

\author{
Do-Hyung KIM*, Soo Jin Yoon, Sang Hyun Jang, Jae Guk KIM, Sung-YeonSohn, Han Na Choi, Jin Ok Kim and SooJoo \\ LEE
}

Department of Neurology, Eulji University Hospital, Eulji University School of Medicine, Korea

Submission: November 07, 2017; Published: December 23, 2017

*Corresponding author: Do-Hyung KIM, Department of Neurology, Eulji University Hospital, School of Medicine, Eulji University, DunsanSeo-ro 95 , Seo-gu, Daejeon 35233, Republic of Korea, Fax: +82-42-611-3858; Tel: +82-42-611-3431; Email: semoxx@naver.com

\begin{abstract}
Spinal cord epidural abscesses can produce atypical symptoms, leading to high rates of delay and misdiagnosis and resulting in increased morbidity and mortality rates. A 63-year-old woman was examined and treated for symptoms of viral meningitis, and exhibited minute clinical improvement. The patient then underwent magnetic resonance imaging (MRI), and a lumbosacral spinal cord epidural abscess was identified. The abscess was treated with antibiotics and surgery. Here, we present this case report demonstrating an unusual presentation of symptoms of spinal cord epidural abscess, which primarily involved pain in regions surrounding the abscess, fever, and neurological deficit.

Keywords: Spinal cord epidural abscess; Central nervous system infection; Cerebrospinal fluid

Abbreviations: CNS: Central Nervous System; MRI: Magnetic Resonance Imaging; CT: Computed Tomography; ESR: Erythrocyte Sedimentation
\end{abstract} Rate; CRP: C-Reactive Protein

\section{Introduction}

Central nervous system (CNS) infections are caused by various pathogens. The most common causes of these infections are viruses and bacteria that cause meningitis; viral meningitis is known to have relatively mild symptoms, whereas viral encephalitis and bacterial meningitis are associated with more severe outcomes. General symptoms for meningitis include headache, fever, nausea, vomiting, and mental deterioration. Cerebrospinal fluid examination is extremely important in the diagnosis of CNS infections. Several measurements obtained during lumbar puncture are used to guide treatment. These include opening pressure, number of cells, protein and glucose levels, and the ratio of glucose in spinal fluid and blood. These metrics can be used to determine the potential pathogen, inform the use of antibiotics and steroids, and determine treatment direction, such as the use of injections or antiepileptic drugs to reduce pressure in the skull [1]. Here, we report a case of suspected viral meningitis that was later identified to be a lumbosacral spinal cord epidural abscess distant from the brain using additional MRI examination, and discuss this case within the context of current literature.

\section{Case Report}

A 63-year-old woman was hospitalized in the division of infectious diseases in the internal medicine department after presenting with fever, and was referred to the neurology department owing to continuous nausea, vomiting, and headache. The patient complained of a fever that increased in intensity from the back to the head, and although the patient was perspiring from the chest, neck, and head, body temperature was maintained at $37{ }^{\circ} \mathrm{C}$. The fever outbreak was accompanied by headache. Coughing caused the headache to worsen and caused ringing. Nausea and vomiting began the day after headache onset, and the intensity of the headache was stable. The patient did not suffer from photophobia or phonophobia; however, partial neck stiffness was detected during physical examination. Although consciousness, orientation, and memory were normal during the nervous system examination, the patient could not concentrate and responded to questions absentmindedly. Articulation was normal, as were motor and sensory functions, deep tendon reflex and cerebellar examination. Blood obtained by the Division of infectious disease exhibited increased Erythrocyte sedimentation rate (ESR) $(39 \mathrm{~mm} / \mathrm{hr})$ and C-reactive protein (CRP) $(8.23 \mathrm{mg} / \mathrm{dl})$. Results for blood, sputum, urine, and stool cultures, and computed tomography (CT) examination of the chest and abdomen were normal. Because CNS infection was suspected, cerebrospinal fluid examination was conducted after the transfer to the neurology department. The fluid was clear, with a pressure of $21 \mathrm{~cm} \mathrm{H}_{2} \mathrm{O}$, white blood 


\section{Open Access Journal of Neurology \& Neurosurgery}

cell count of $1400 / \mathrm{mm} 3$ (neutrophils $26 \%$, lymphocytes 58\%), less than 5 red blood cells, $62.2 \mathrm{mg} / \mathrm{dL}$ protein, and $52 \mathrm{mg} /$ $\mathrm{dL}$ glucose. There was $123 \mathrm{mg} / \mathrm{dL}$ of glucose in the peripheral blood, and ratio of glucose in cerebrospinal fluid/blood serum was 0.42 . Based on these results, the patient was diagnosed with viral meningitis and treated with intravenous injection of an antiviral drug (Acyclovir). However, after 4 days of treatment, symptoms including headache, nausea, and vomiting persisted, as well as a fever of $37-38{ }^{\circ} \mathrm{C}$ that worsened at night, were observed. Although a brain MRI was normal, considering the clinical presentation of symptoms and the lack of response to antiviral treatment, tuberculosis meningitis was suspected and the patient was additionally treated with Rifampicin, Isoniazid, Pyrazinamide, and Ethambutol. However, fever, headache, and nausea persisted after 1 week of treatment.

Bacterial and fungal culture tests of cerebrospinal fluid were negative. Tubercular bacillus and fungi smear tests, culture tests, and adenosine deaminase level (6.9IU/L)were normal. Polymerase chain reaction (PCR) tests on cerebrospinal fluid for herpes simplex virus, herpes zoster virus, cytomegalovirus, and Epstein-Barr virus, as well as for tubercular bacillus, were negative. Hepatitis B surface antigen (HBsAg), Human Immunodeficiency Virus (HIV), and Treponema pallidum nontreponemal tests (venereal disease research laboratory) on blood were also negative. After 8 days of additional administration of anti-tubercular drugs, blood ESR and CRP levels continued to increase, although white blood cell count was normal, and symptoms did not subside. Hence, all treatments of anti-tubercular and antiviral drugs were halted and the patient was treated with intravenous injection of third-generation cephalosporin, vancomycin, and ampicillin in accordance with treatment of a CNS bacterial infection. The day after initiation of antibacterial treatment, sweating and cervical rigidity persisted, but fever was subsided and the patient was in a stable condition; perspiration gradually improved. However, after one week of antibacterial treatment, vancomycin administration was halted and the patient was treated only with third-generation cephalosporin and ampicillin. This led to recurrence of sweating and fever, therefore cerebrospinal fluid examination was repeated, and an improvement from pre-treatment conditions was observed. However, clinical symptoms including fever re-surfaced. An interview on the patient's medical history was repeated, during which the patient reported no previous abnormalities in lower extremities, sensation, or urination; however, the patient complained of back pain that began several months prior. The patient had received treatment for back pain in another hospital one week before the fever outbreak and had received continuous pain relieving injections for up to 3 days before hospitalization. MRI examination was conducted on the spine, in which an epidural abscess was identified in the spinal cord, accompanied by arachnoiditis and caudaequina neuritis located bilaterally between the $5^{\text {th }}$ lumbar and the $1^{\text {st }}$ sacral vertebrae, osteomyelitis in the left $1^{\text {st }}$ and $2^{\text {nd }}$ sacral vertebrae, and leptomeningitis in the thoracolumbar region of the spinal cord (Figure 1).

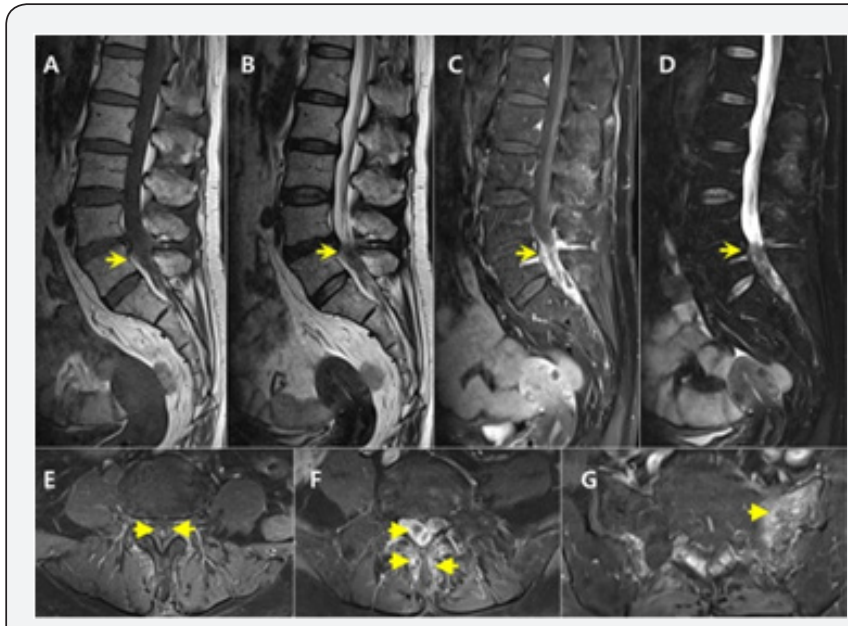

Figure 1: Sagittal T1-weighted image of lumbosacral spine (A), T2-weighted image (B), T1-weighted fat saturated gadolinium enhancement MRI (C), T2-weighted Dixon sequence MRI(D). Heterogeneous contrast enhancement along the epidural surface located inner side of spinal canal of the $5^{\text {th }}$ lumbar and the rear 1 st sacral vertebrae, suggesting epidural abscess accompanied by arachnoiditis, and local irregular thickening were observed. T1-weighted fat saturated gadolinium enhancement MRI (E, F, and $\mathrm{G}$ ) taken in the axial direction of lumbosacral spines how abnormal contrast enhancement on the surface of multiple nerve roots of cauda equine, and local irregular and heterogeneous contrast enhancement suggesting spinal osteomyelitis in the left outer bone marrow of the $1^{\text {st }}$ and the $2^{\text {nd }}$ sacral vertebrae $(G)$.

\section{Result}

The patient was diagnosed with epidural abscess accompanied by arachnoiditis and leptomeningitis, and abscess removal surgery was performed in collaboration with the neurosurgery department. The patient received injections of antibiotics (vancomycin, cefoperazone, and sulbactam) for 2 weeks, which relieved headache, fever, nausea, and vomiting. The patient was then discharged.

\section{Discussion}

Spinal cord epidural abscesses are caused by pyogenic infection in the epidural space of the spine. The abscess can directly exert pressure on the spinal cord, conus medullaris, or cauda equine, or exert pressure on surrounding blood vessels that supply these structures [2], reducing blood flow and resulting in various neurological symptoms [3]. According to previous reports, the number of spinal cord epidural abscess patients is $2.5-3.0$ per 10,000 [4] and the morbidity rate is gradually increasing, presently accounting for $10 \%$ of protopathic spinal cord infections [3]. Morbidity associated with spinal cord epidural abscesses is increasing in people over 60 years of age. The prevalence is higher in males, $30-50 \%$ of patients suffer from permanent neurological injury, and the mortality rate is $10-20 \%$ [3]. In general, thetriad of spinal cord epidural abscess symptomsis 1) back pain in regions surrounding the 
abscess (75\% of spinal cord epidural abscess patients), 2) fever (about 50\%), and 3) neurological deficits (about 33\%). These symptoms are atypical and patients rarely suffer from all three, leading to difficulty in diagnosis. This results in delayed or misdiagnosis, poor prognosis, and increased mortality rate [4,5]. Spinal cord epidural abscess patients often have the following risk factors: diabetes, intravenous drug use, alcohol addiction, HIV infection, degenerative arthritis, spinal osteomyelitis, endstage renal failure, are undergoing immunosuppression therapy, have had a recent injury and surgery history. Further, invasive spinal procedures conducted to regulate pain represent a risk factor: $14-22 \%$ patients suffer from an abscess after receiving spinal surgery, percutaneous spinal procedures such as epidural steroid injection, and nerve block procedures $[3,4]$. The patient in our case suffered back pain in the past and underwent a percutaneous procedure prior to hospitalization. Her symptoms during hospitalization and at transfer to the neurology department (fever, continuous nausea, vomiting, and headache) strongly suggested meningitis, thus the diagnosis of spinal cord epidural abscess was difficult. In general, spinal cord epidural abscess is caused by Staphylococcus aureus, Staphylococcus epidermidis, Escherichia coli, Pseudomonas aeruginosa, and Mycobacterium tuberculosis, as well as rare fungi [3]. However, our patient tested negative in all culture, gram staining, and PCR tests on cerebrospinal fluid and blood.

According to previous reports, only half of patients suspected of spinal cord epidural abscess test positive in blood culture tests [4], most gram staining results are negative even when the cerebrospinal fluid test suggests infection, and culture tests are positive in less than $25 \%$ of the patients [5]. Thus, in many cases, such as ours, it is difficult to identify the pathogen. Our patient, like most with spinal cord epidural abscesses, showed increased ESR and CRP [3] (although there was no increase in white blood cell count) in repeated blood tests; both of these decreased upon antibiotics treatment. In spinal cord epidural abscess diagnosis, it is also important to identify the presence and location of the abscess through gadolinium contrast enhancement MRI on the whole spine $[3,6]$.

Our patient did not show any abnormality in brain MRI; however, the abscess was identified in a spine MRI, leading to the diagnosis. Treatment of the abscess requires both internal medical and surgical approaches; surgical drainage and widescale surgical debridement must be conducted as soon as possible upon diagnosis (unless surgery is contraindicated), and antibiotics must be administered according to the infection as in all other CNS infectious diseases. In patients suspected of bacterial spinal cord epidural abscess accompanied by spinal osteomyelitis, as in our patient, vancomycin is used to treat gram-positive bacteria and third-generation cephalosporin or aminoglycoside antibiotics are used to treat gram-negative bacteria for 4-6 weeks [3,4]. In this patient, while antiviral and anti-tubercular drug treatment did not improve clinical symptoms such as fever, antibiotics including vancomycin resulted in rapid improvement. Worsening of symptoms upon cessation of vancomycin treatment suggested gram-positive bacteria as the cause of the spinal cord epidural abscess.

Spinal cord epidural abscess essentially requires early diagnosis and treatment, therefore detailed interviewing as well as physical and neurological examinations need to be conducted. As well as having aforementioned risk factors, patients exhibit the triad of symptoms (back pain, fever, neurological deficits) of spinal cord epidural abscess. If patients also exhibit elevated ESR and CRP levels, gadolinium contrast enhancement MRI should be conducted on the whole spine. However, lumbosacral spinal cord epidural abscesses located far from the head could also show typical clinical symptoms of meningitis and spinal fluid test results. If meningitis treatment does not lead to any improvement in symptoms, the possibility of spinal cord epidural abscess should be considered.

\section{Conflict of Interest}

All authors declare no conflicting interests and none of them are supported/funded by and drug company.

\section{References}

1. Stoeter DJ, Michael BD, Solomon T, Poole L (2015) Managing acute central nervous system infections in the UK adult intensive care unit in the wake of UK encephalitis guidelines. J Intensive Care Soc 16(4): 330-338.

2. Feldenzer JA, McKeever PE, Schaberg DR, Campbell JA, Hoff JT (1988) The pathogenesis of spinal epidural abscess: microangiographic studies in an experimental model. J Neurosurg 69(1): 110-114.

3. DeFroda SF, DePasse JM, Eltorai AE, Daniels AH, Palumbo MA (2016) Evaluation and management of spinal epidural abscess. J Hosp Med 11(2): 130-135.

4. Bond A, Manian FA (2016) Spinal epidural abscess: a review with special emphasis on earlier diagnosis. Biomed Res Int 2016(2016): 1614328.

5. Darouiche RO (2006) Spinal epidural abscess. N Engl J Med 355(19): 2012-2020.

6. Pradilla G, Ardila GP, Hsu W, Rigamonti D (2009) Epidural abscesses of the CNS. Lancet Neurol 8(3): 292-300. 
This work is licensed under Creative Commons Attribution 4.0 Licens

DOI: 10.19080/OAJNN.2017.06.555695
Your next submission with Juniper Publishers will reach you the below assets

- Quality Editorial service

- Swift Peer Review

- Reprints availability

- E-prints Service

- Manuscript Podcast for convenient understanding

- Global attainment for your research

- Manuscript accessibility in different formats

( Pdf, E-pub, Full Text, Audio)

- Unceasing customer service

Track the below URL for one-step submission https://juniperpublishers.com/online-submission.php 